Alignment: /Users/garethweedall/Desktop/igll.fas

Seaview [blocks=70 fontsize=10 A4] on Fri Nov 5 15:25:11 2010

DS571197_51170-71066 DS548835_12593-32400

DS571197_51170-71066 DS548835_12593-32400 139

DS571197_51170-71066 DS548835_12593-32400 208

DS571197_51170-71066 DS548835_12593-32400

277

DS548835_12593-32400

346

DS571197_51170-71066 DS548835_12593-32400

DS571197_51170-71066 DS548835_12593-32400

DS571197_51170-71066 DS548835_12593-32400

DS571197_51170-71066 DS548835_12593-32400 622

DS571197_51170-71066 691

DS571197_51170-71066 DS548835_12593-32400

DS571197_51170-71066 DS548835_12593-32400

829

DS571197_51170-71066 DS548835_12593-32400

898

DS571197_51170-71066 DS548835_12593-32400

DS571197_51170-71066 DS548835_12593-32400

1036

DS571197_51170-71066 DS548835_12593-32400

1105

DS571197_51170-71066

DS548835_12593-32400

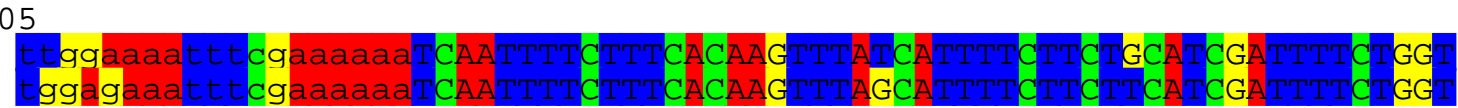

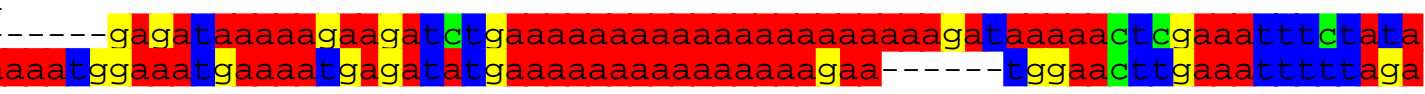
70

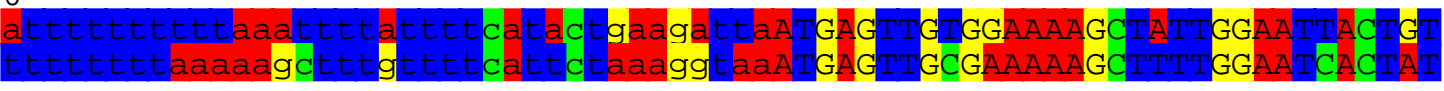
9

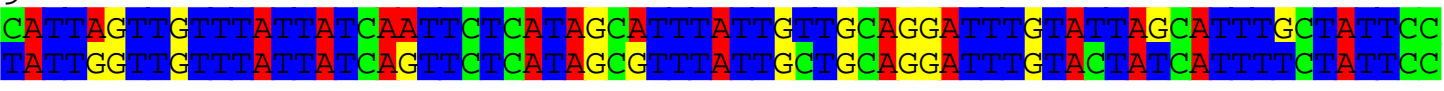
ATCATATGTGATAGAAAGATCTTGAACGTAACGTCATGGATACAAAAGGGTTCTTGAAGGATG
ITCGIATGIGTATAGTAAAGATCTTGAAAGTTAGIGACATGIGATACAGATGGIGTACTTGAAGGATG GAG GATTTCATATAAACAGGTATTATTTGGGTACTAAATAAATTTTGTTAGTGTGCTTTGG
GAAATTICATATAAGICAGGTATTATTTGGGTATTAAATATATTTIGTAGCGATCTTTGG GATTGCAGTCTTTTCTTTCATTCCACTTGTTGGGTTATTAGATTTCATTCTTGGGATATTTACAAGTTG
GATTGCTGTCTTATCTTTCATTCCAGTTGTTGGGTGTTTGATTTTATTCTTGGATAATTACAAGTTG 15

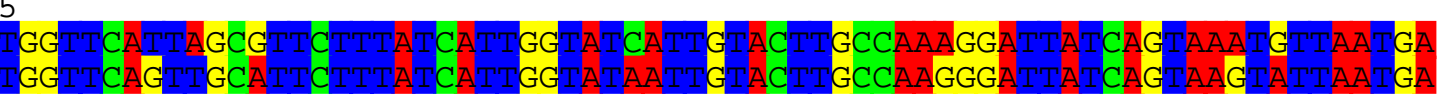
84 ATTAGACAATGAAAAAAATATGTTTGAAAAACAAAAAGATGTGTTTGGATGATTTAATTGTC
ATTAGATGAAGAAAAAAAATATGTTTGAAAACAGAATAGATGTTGTTGGATGTATTCATTGTC 553 AGAATGACATACACAGATAAACTGGTAATACAACAATGTCAGATGATGTAATGAAGTGCTGT
AGAATGTACATATACAGATAAACIGGTCAATTACAACAATGTCAGATGATITAATGAAGCIGTTGI 22

CACTCCAGCAGAACACTCTGTCAAAGTTATTGGTCTATGCTAATTIAGGTTCAGGAATGATGGGGG
CAACCCAGCAGAACATICCGTCAGAGTAICGGTICTATGCTAATTGTAGGTCAGGGATATGGGTG 91

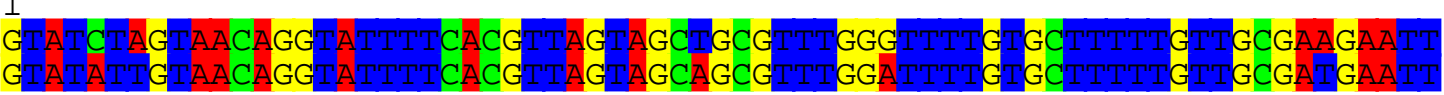
760

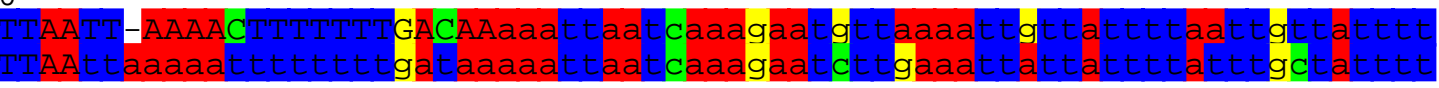

\title{
cattatct qctgattaqaaaaatgtattaatotaacatt---ctctacacttcac
}

cgttatetgttaaatagaaaact - tattattgtgaataataaccgttaatcatttat--ttatattt 98

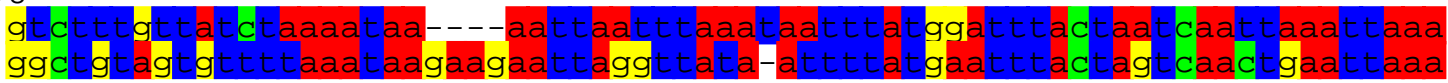
967

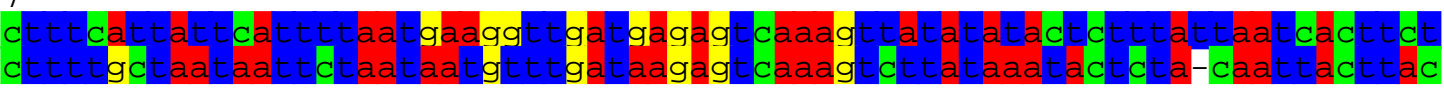
attatatcaataactattatgaacaaatttaaataqaaa---------atattaaaatgaaaad
actgtatcaacaagttt attaagaaactaattagaaagttaaattataaaaattaaaaact 1174 DS571197_51170-71066 CTAATGGTTAATGATGGGATTTAACCACTCTGTTATAAACCAATGTAGCAAAGAACAAAATCACAA DS548835_12593-32400 CTAATGGTCAATGAIGGGAATTAACCACICTATTATAAACCAATGIAGCAAAGAATAAATCACAA 


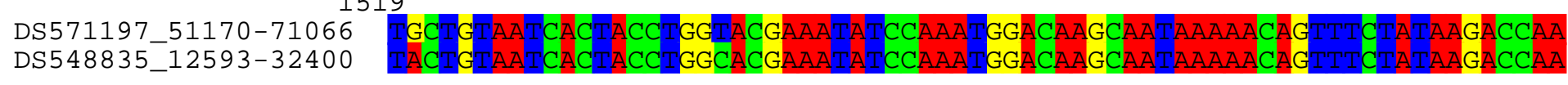

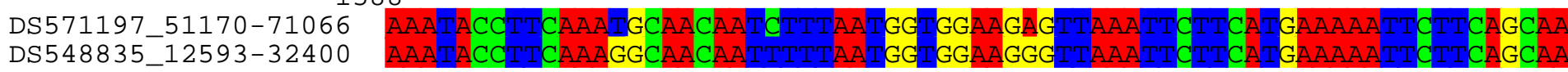

1657

DS571197_51170-71066 CAATTIGTCCAGCTIGGATAAATIGGGCAATTAAAATCATAAGAATACCCATIGCTGTTIGGATCCAG DS548835_12593-32400 CAATIIGICCAGCIIGAATAAATIGGGCAATTAAAAICATAAGAATACCCAITGCIGITIGGAAICCAG

1726

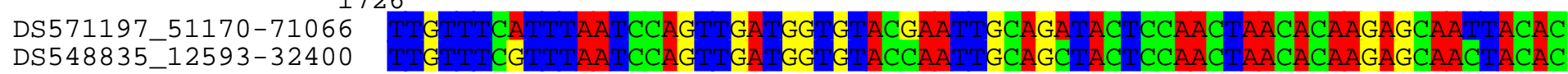

1795

DS571197_51170-71066 DS548835_12593-32400

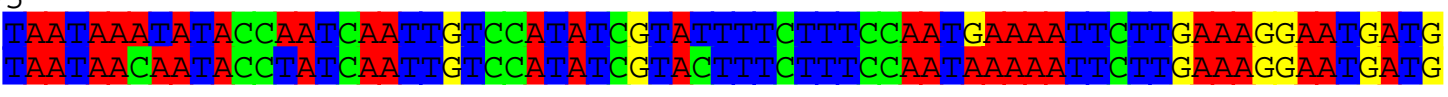

1864

DS571197_51170-71066 DS548835_12593-32400

DS571197_51170-71066

DS548835_12593-32400

\section{2}

DS571197_51170-71066 DS548835_12593-32400

2071

DS571197_51170-71066

DS548835_12593-32400
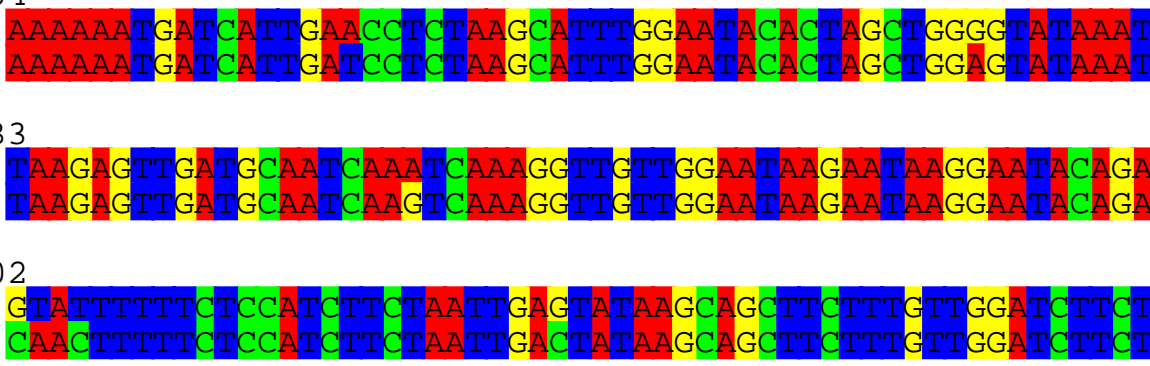

TGGAATAGAATAAGGATACAGAGGCTIGTTTTICAG
TGGAATAAGATAAGGATACAGAAGCTIGTITICAGG

TAAATAATCAA
IATATAATCAA

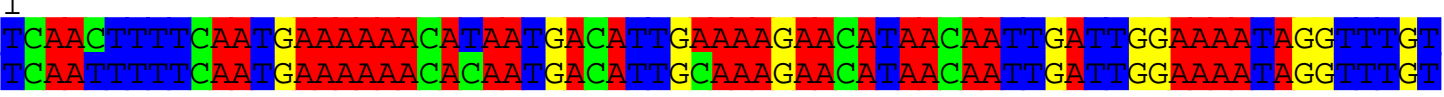

2140

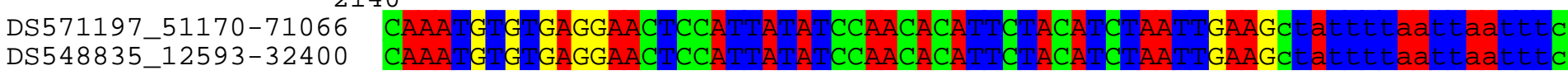
2209

DS571197_51170-71066

DS548835_12593-32400
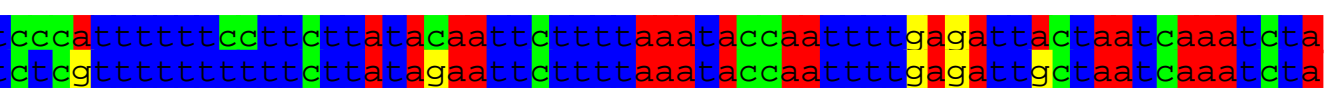

2278

DS571197_51170-71066

DS548835_12593-32400
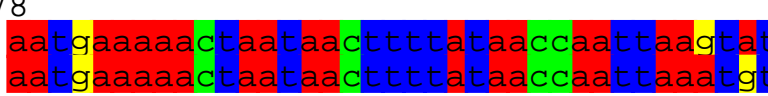

2347

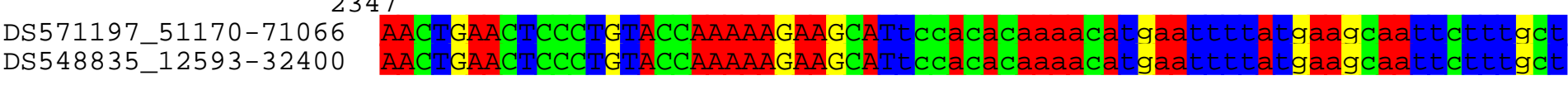
2416

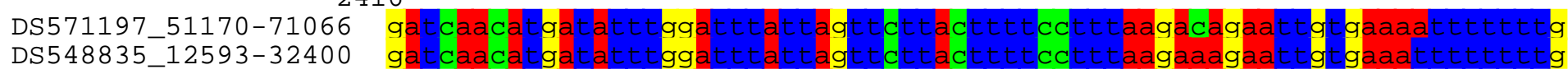

2485

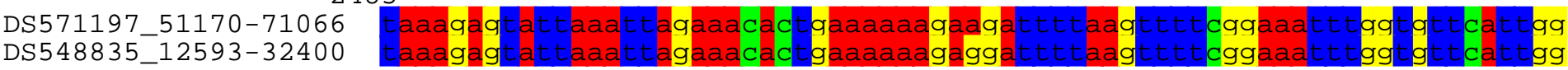

2554

DS571197-51170-71066 aaaatcctaccaaccaacgaqqattaqacattaaqaaattaCTAATTGTTTAATACACCAACAACAT DS548835_12593-32400 aaaagtcct-accaaccaacaggattagacataagaaactaCTAATTATIAAAIGCACTAACAACGI 

DS571197-51170-71066 C DS548835_12593-32400

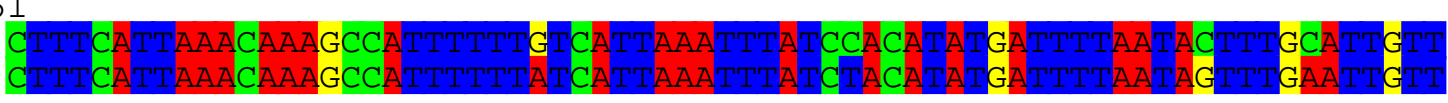

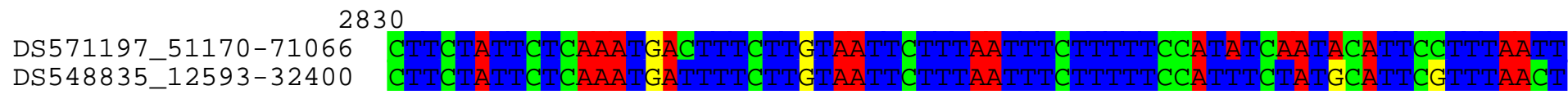
2899

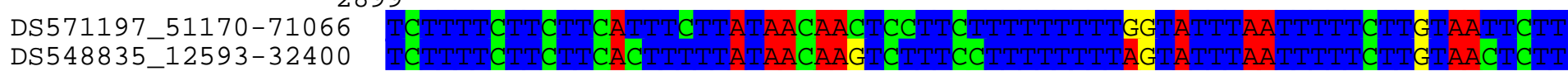

2968

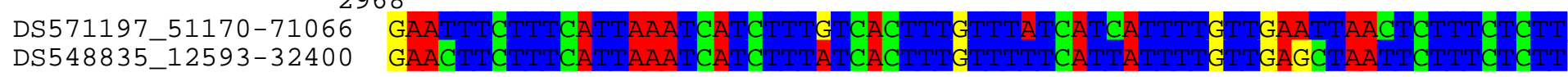
3037

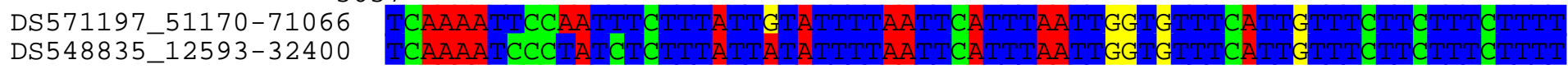

3106

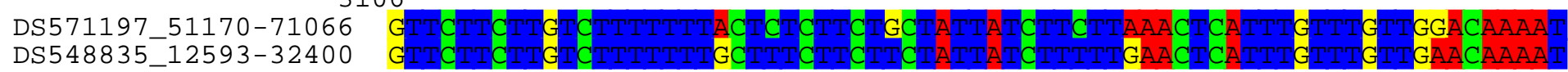
DS571197 $51170-71066$ A DS548835_12593-32400

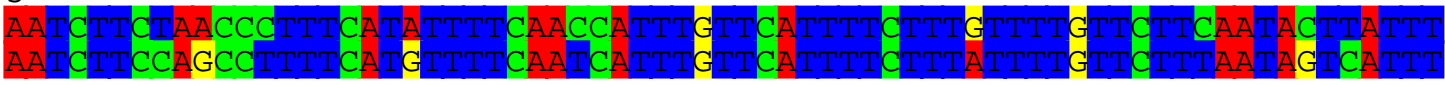
3244

DS571197_51170-71066 DS548835_12593-32400

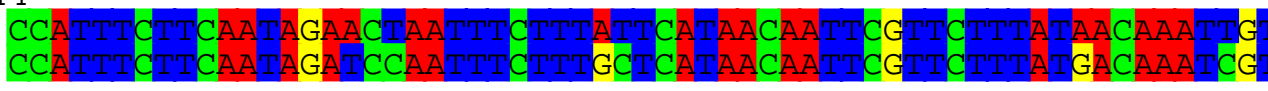

DS571197_51170-71066 DS548835_12593-32400

\section{2}

DS571197_51170-7106 DS548835_12593-32400

3451

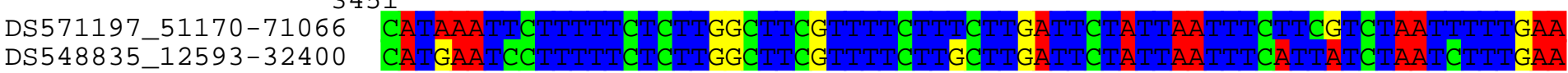
DS571197 51170-71066 DS548835_12593-32400

3589

DS571197_51170-71066 DS548835_12593-32400
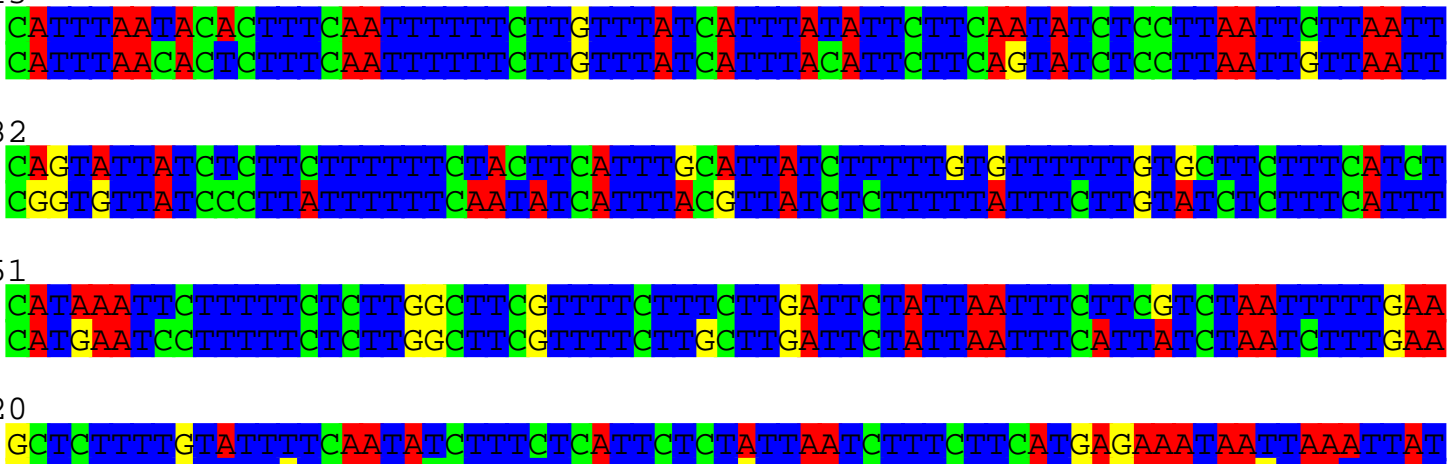

DS571197_51170-71066

DS548835_12593-32400

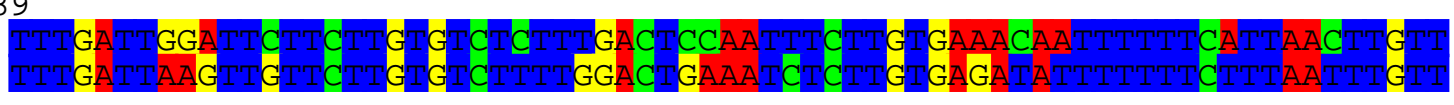

3658

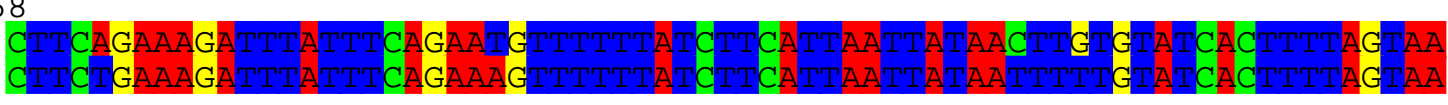

3727

DS571197_51170-71066

DS548835_12593-32400

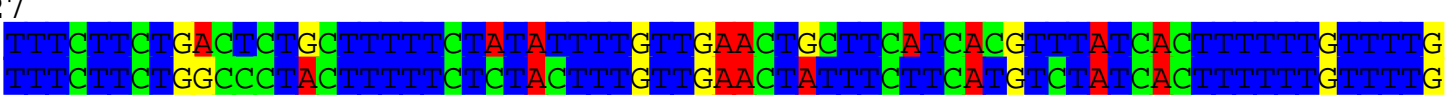

3796

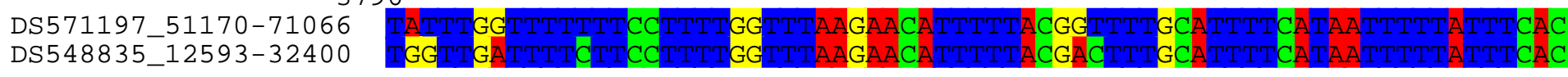

3865

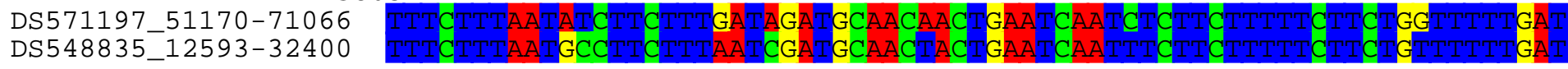

3934

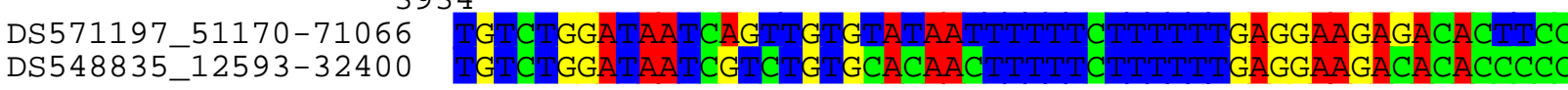

4003 

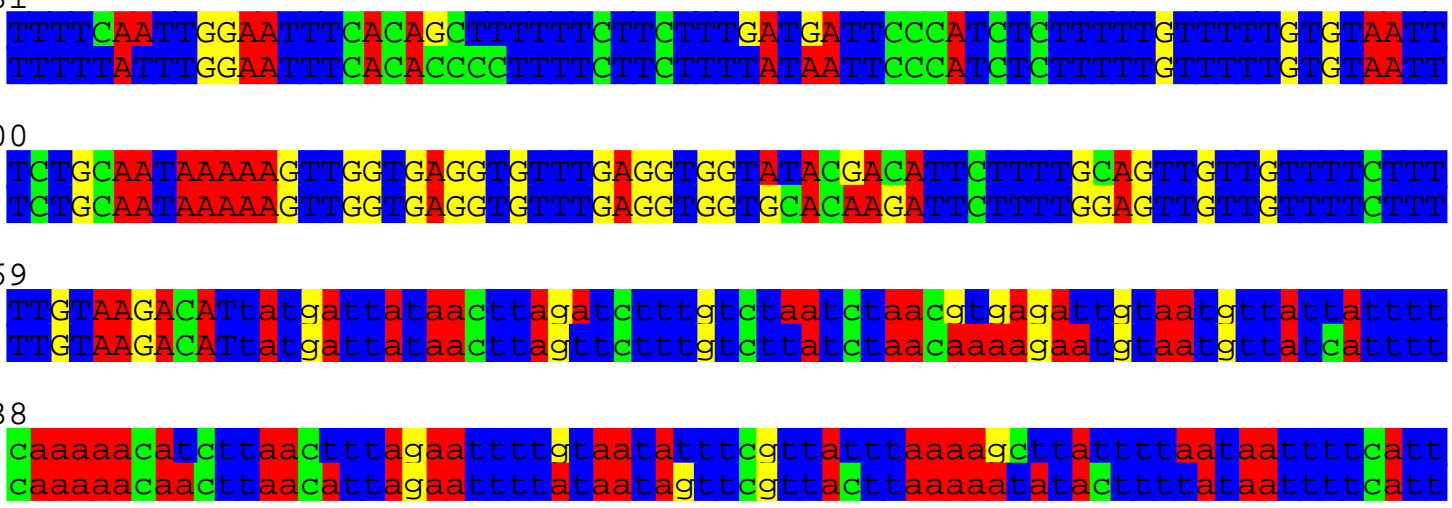


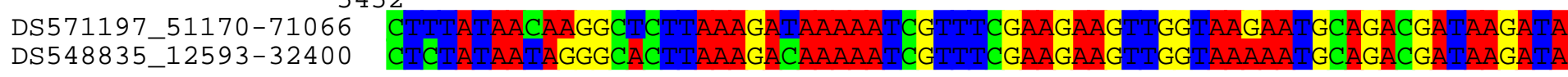
5521

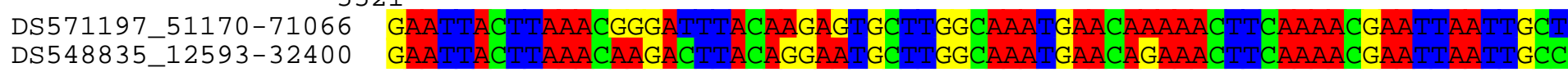
5590

DS571197_51170-71066 GAAAAGGAGGACATGAAAAAGCTGAATAGAATTAAAACAAAAAACAGTCGGAAGTAAAATTCTTACT
DS548835_12593-32400 GAAAAAGAAGACATGAAAAGCAGAAAAAGAATIAAACAAAAAACTATGGAAGAAAGICTIACI

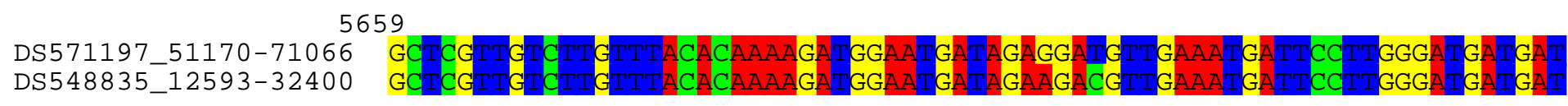
5728 DS571197_51170-71066 TICGATGCTTCTGCAGATGTATTAGAAAAATTAAAAAGACACAAACAAACATAATCACTGAAAGAAAA
DS548835_12593-32400 TICGATGCTICIGCAGATGITATTAGAAAAATTAAAAAGACACAAACAAACATAGICACIGAAAGAAA

5797

DS571197_51170-71066 GAAATTICAGAAAATCTTAAAAATACTCAACCTCAATCTCAATCTAAGTTACCTCTTTCAAATGAACAG DS548835_12593-32400 GAAATICAGATAICITAAAAACAGICAGCCICAAICICAG------TTACCICTTICAAATGAACAA 5866 DS571197_51170-71066 CCAACTTCATTTAATACCACTACTATCCGICCAAAAAATTTTCAGTTAGTCAAATTCAAAAGGACCTT
DS548835_12593-32400 ICAACCICATTIAATACAACI---AICCICCAAAAAATTTTTAGTIAGACAAATCAAAAGGACCTT

5935

DS571197_51170-71066 GAAAAGTTGAAAAAAGGTCGAGGAATAATTAAAATTACACAATCACCAAATCATTTAGAAAAGAAA
DS548835_12593-32400 AAAAAATTGAAAAACGGCIGACGACATTATCAAAATTACACAACACCAAAICACTITGAAAGAAA 6004

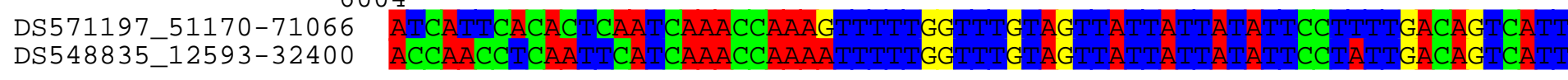
6073

DS571197_51170-71066 DS548835_12593-32400
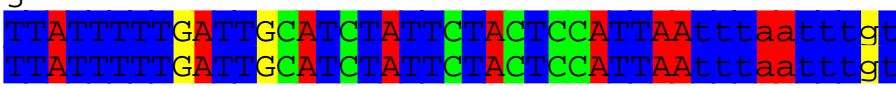
ttaq
tcag

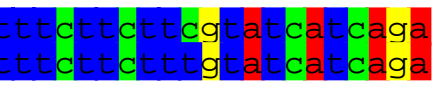
6142

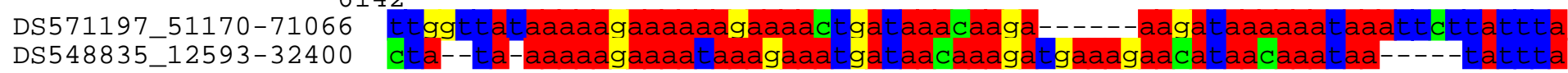
6211

DS571197_51170-71066 aaaatagaactgacaaaaattcattgtgcaaaaaacacaat taatttt g-aaaacaaaatta-
DS548835_12593-32400 aaaataaaaataacataaatttgttaatc--aaaaacacaactgattactataaaatgaatattaa

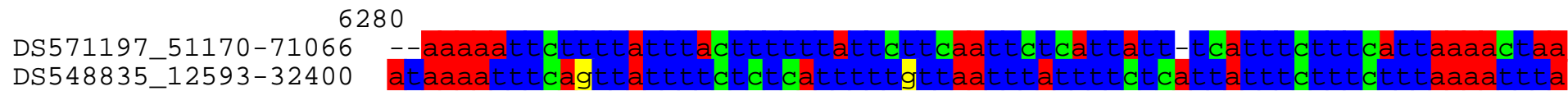
6349

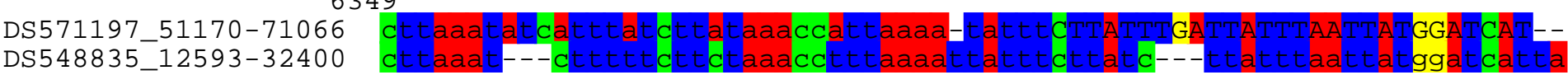
6418

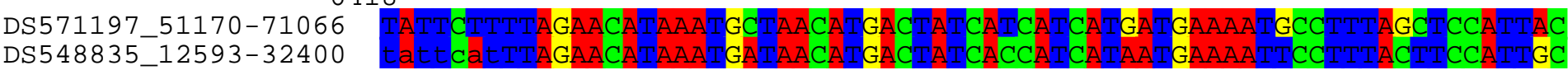
6487

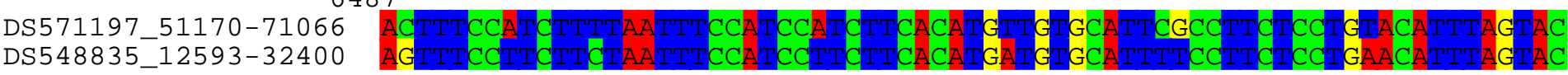
6556

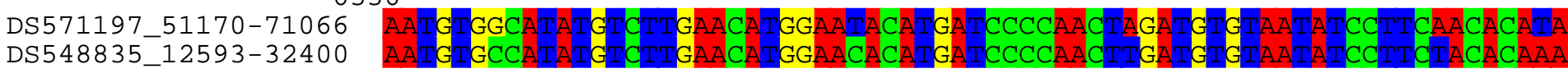
6625

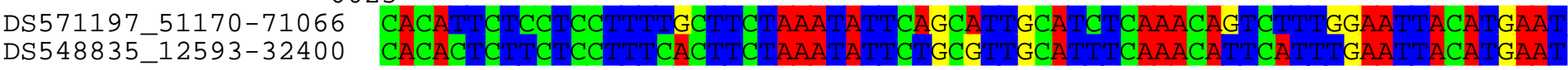
6694

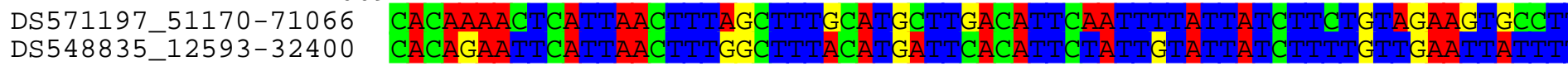




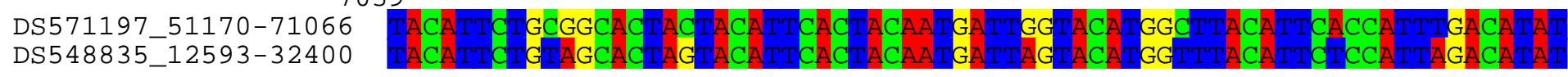

7108

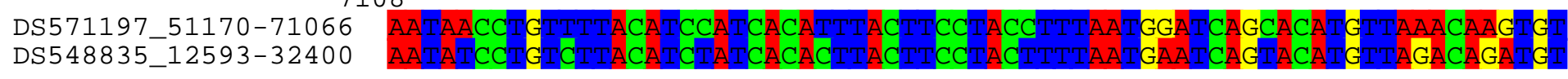
7177 DS571197_51170-71066 CTTTATCTGAACATGTTGCACATTTATCATCACATTTAGCACACCCATT-------------------CT
DS548835_12593-32400 CTTGATCTGACAAGICCACATTCAGGATACATTTAGCACATCCATTATTTTATTACCTTCTCCCI

7246

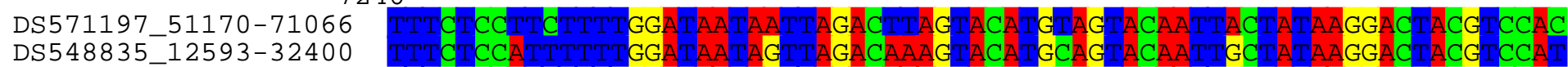

7315

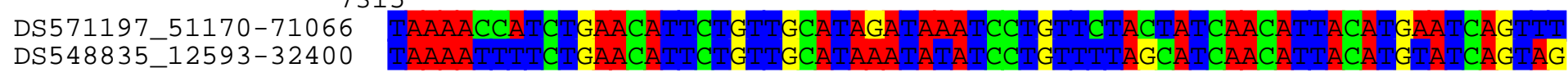

7384

DS571197_51170-71066

DS548835_12593-32400

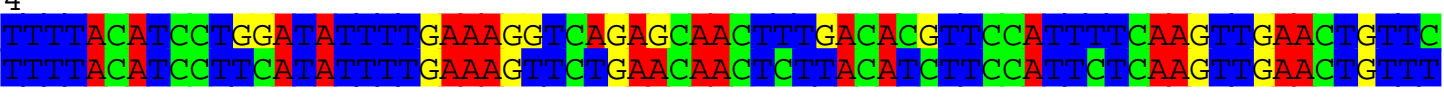
7453

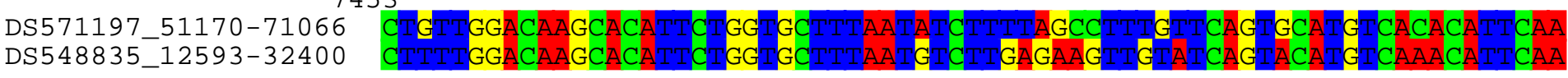
7522

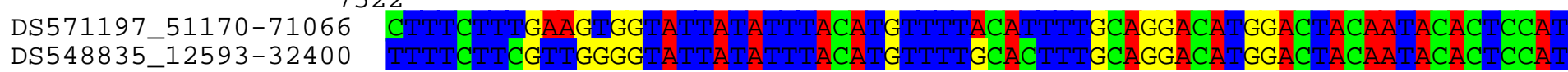

7591

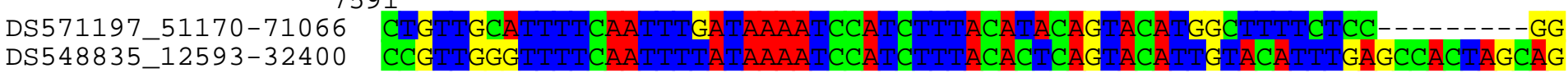

7660

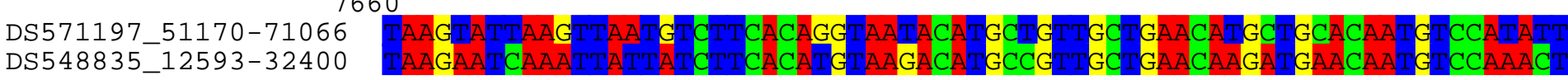
7729

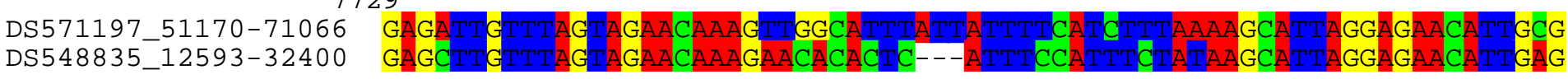

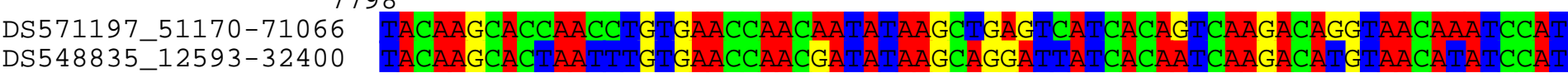
7867

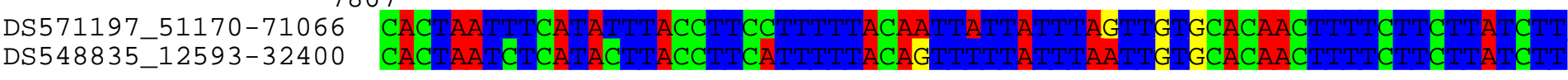

7936

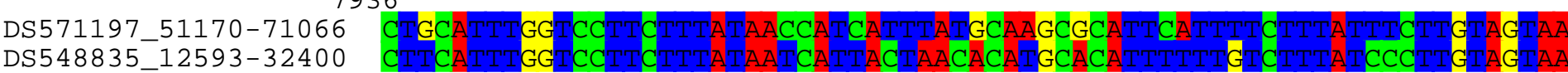
8005

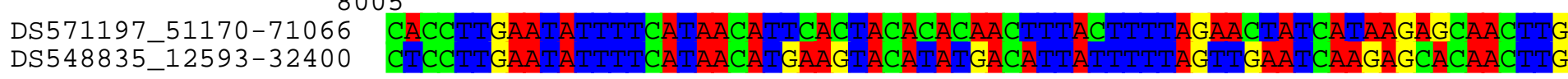

8074

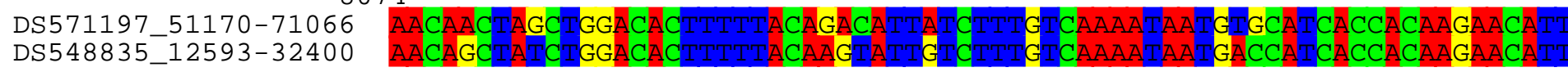




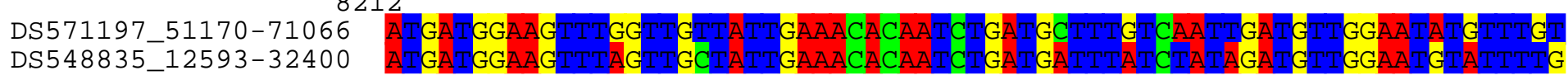
8281

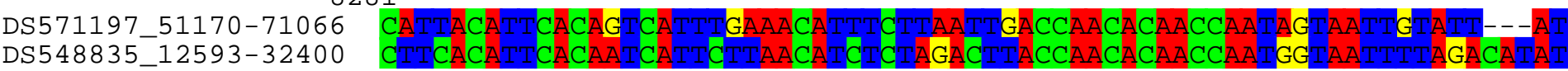
8350

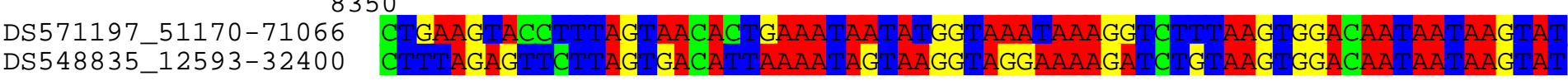
8419

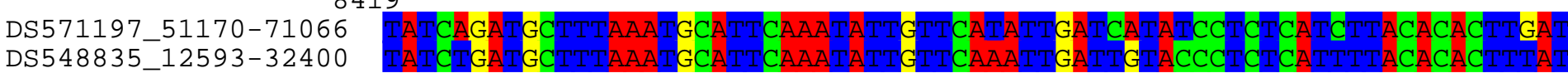

8488

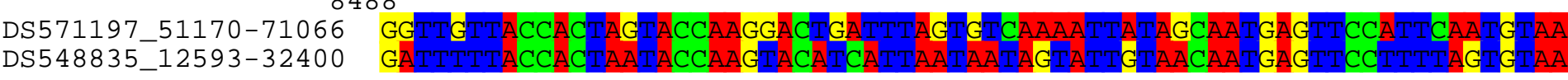
8557 DS571197_51170-71066 GTCCATTCTCACATTIATAACAAATAG ATCATCATCACTACATAAAAACATCCTGGATTIGCATG
DS548835_12593-32400 GICCATICICACAITIATAACAAATAGIAICATCAICACTACAIGACAAACAICCIGGAAGTIACAIG 8626

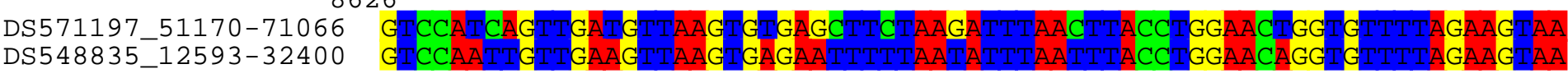
8695

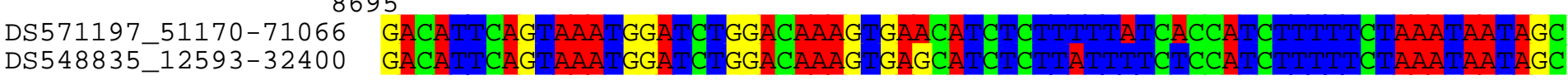
8764

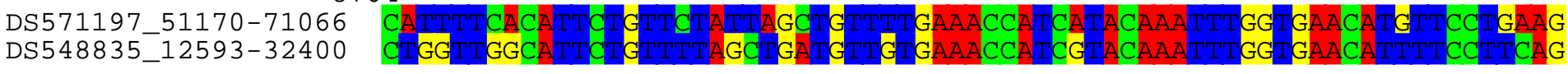
8833

DS571197_51170-71066 DS548835_12593-32400

\section{TTG} TAAGAATATCCATTATCACATTTIGTACA
IAGGAAATAICCATIATCACATTITGCACA ICTICIGTTGGTGAGATAAACGACAAT
ICITAGICAAAGAGACAACGACAAI 8902

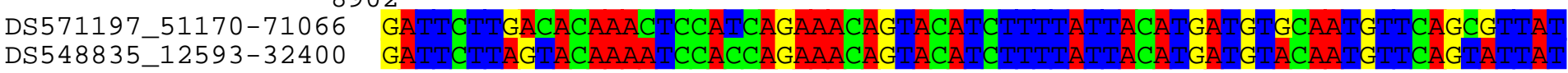
8971

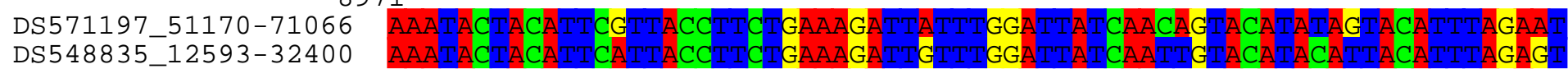
9040

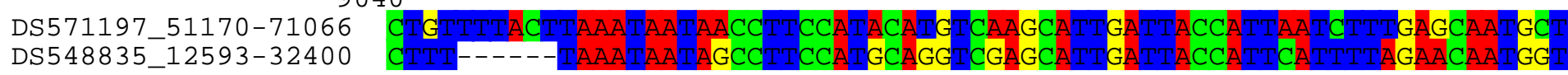
9109

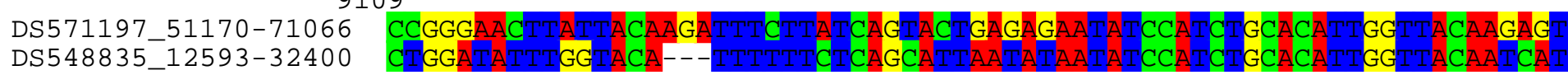
9178

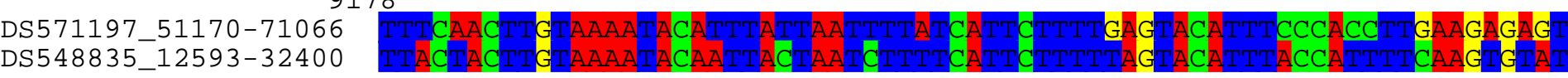
9247

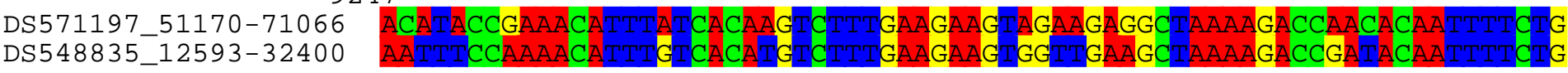
9316

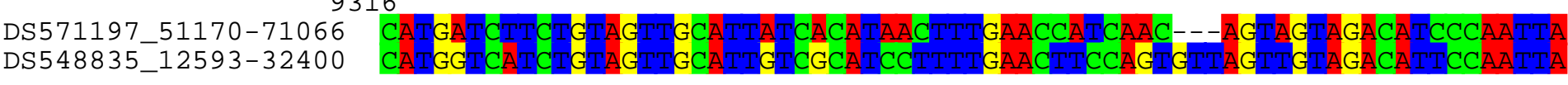
9385 DS571197_51170-71066 TACATTCTCCACATTT--MGAATCTTCATCAAAAGACATGATCACAAATGTTTACATTGTTTTTGC DS548835_12593-32400 IACATICTICACATTIAGGGCACIGICTITAGAAAGACAAG IICACAATIAICAACACCATTTTIAC 9454

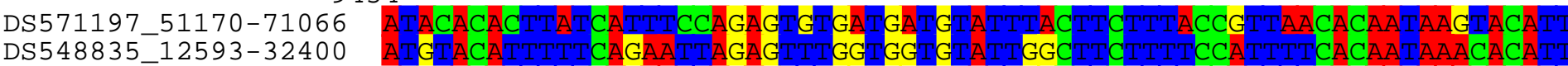



11110 DS571197_51170-71066 aaqaagttgcaattaqaqaaagttaaqtatattgttactcttattcttttta--aaacattctctcta
DS548835_12593-32400 aagaaattccaatcagagaaagttagg--cattatgactttcatcctctttaacagacatagagaaaa 11179

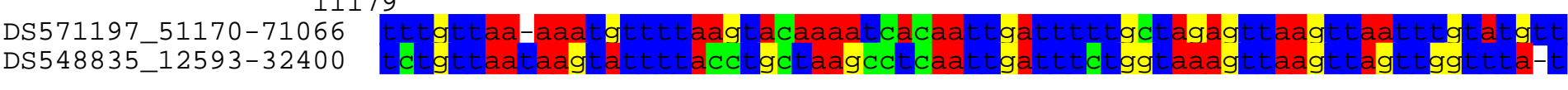
11248 DS571197_51170-71066
DS548835_12593-32400 tettatttggtttaaatttattcaaagaagaatt-tgatgaatgaaaataaaagaattgacttgtta
tettatttgtcttaagattattcaaagaagaattctgatgggtgataataaaagaatcgatt atta

11317

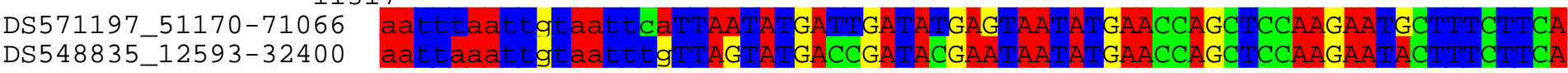
11386

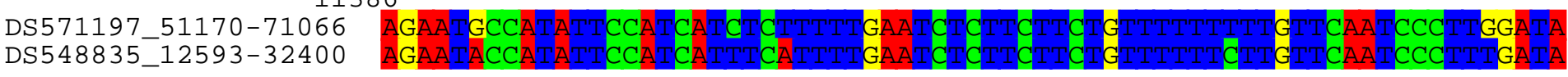
11455

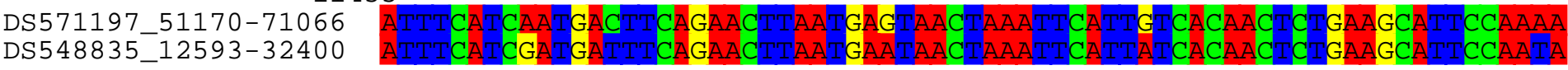
11524 DS571197_51170-71066 DS548835_12593-32400 Mim 11593

DS571197_51170-71066 DS548835_12593-32400
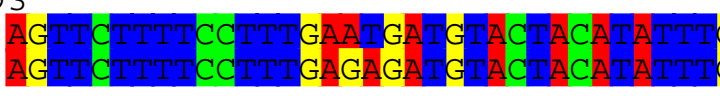
GCCCCTCTACTICD
GCCCTICTACTICI

11662

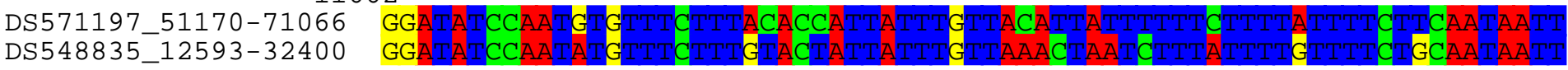

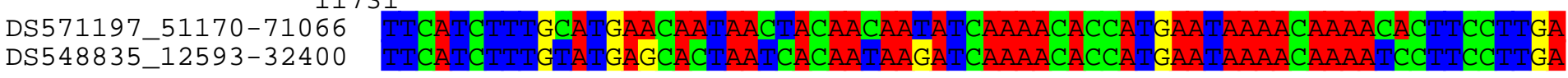
11800 DS571197_51170-71066 DS548835_12593-32400

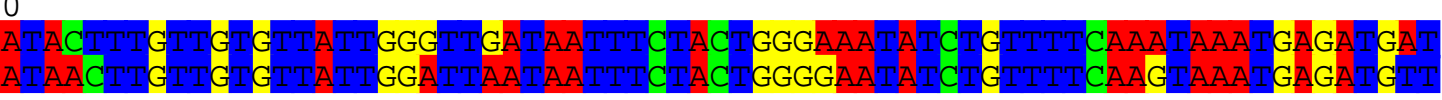
11869 DS571197_51170-71066 DS548835_12593-32400

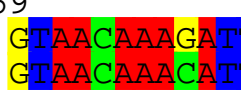
GTTTTTCTCCCCAATGTTTATCCGCAACAACTTCCTTCTTGTTTCCACTTTTT
GTTTTTCICCCCAGTGTTTATICCACAACAACTTCCTTCICGATTTCTACTTCTT

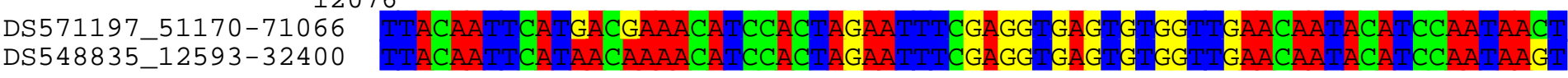
12145 DS571197_51170-71066 GAGTTAAATGGAATACCTTTCTATGACTTTTGCTTTAATTCAATGGTTGTAAATAGATGACCCA
DS548835_12593-32400 GAATTAAATGATATACCTTTCTATGACTTTIGCTTTGATTCAATGGCIGIAAAATAGATGGCCA 


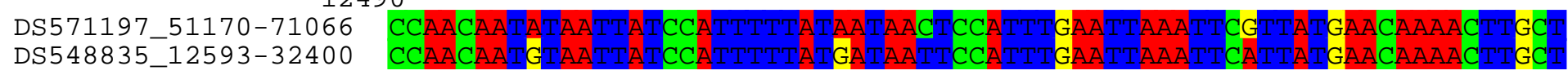
12559

DS571197_51170-71066 DS548835_12593-32400

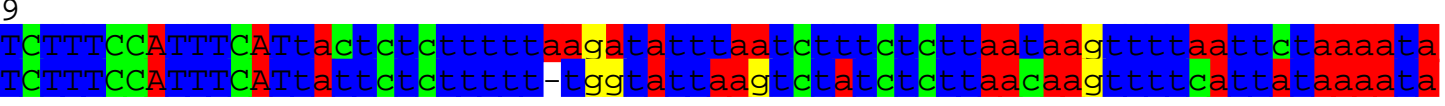

12628

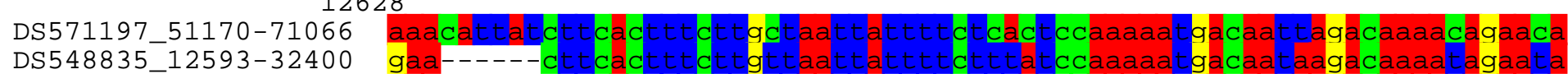

12697

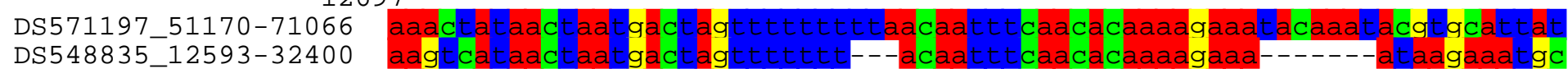
DS548835_12593-32400 aca 12766

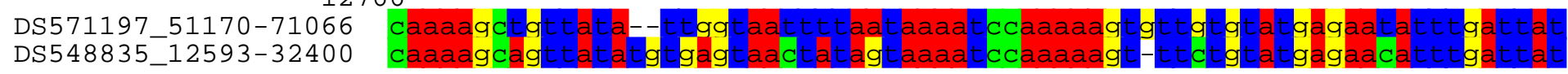
12835

DS571197_51170-71066 DS548835_12593-32400

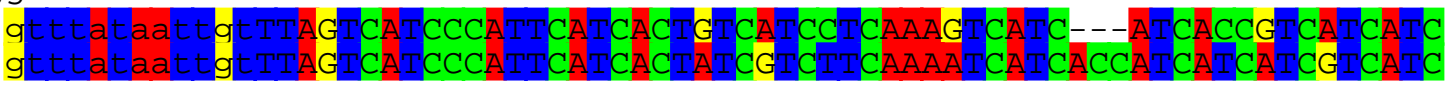

12904

DS571197_51170-71066

DS548835_12593-32400

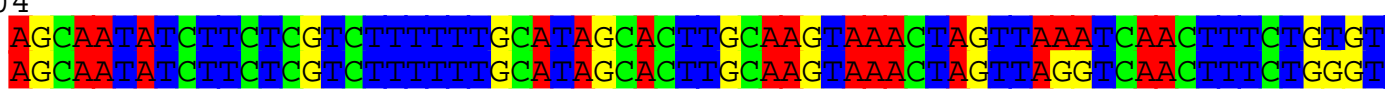

DS571197_51170-71066

DS548835_12593-32400

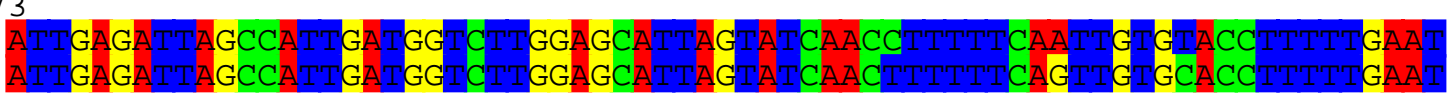

13042

DS571197_51170-71066 TTGGTAAACAAGAAGGGCAGCTGGTGCGCTGGAGCAGCAGGAACTCCATGACTTCCTCTAGAGG DS548835_12593-32400 IIGAICIAATAACGAAGGGCAGCIGGIGCGGGIGG GCAGCAGGGGCICCAIGACTICCICIAGGAGG

13111

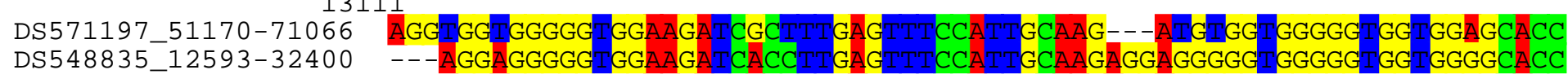

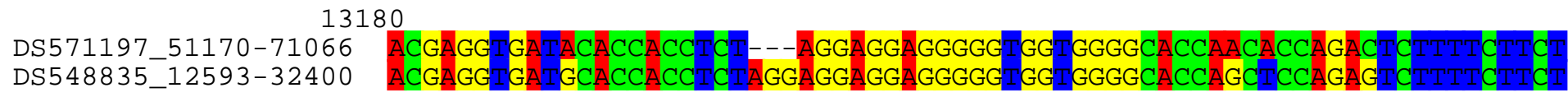

DS571197_51170-71066 TGGAGGATAGGTTTGGGGACTTCCAGATGGTCTTTGAGGTGCTGATTGAGCAGGACGAGTACCAAG DS548835_12593-32400 IGGAAGGCAGGITIGGAGCACTICCAGATGTCTTTGAGG GCIGATIGGGCAGGACGAGCACCAAG

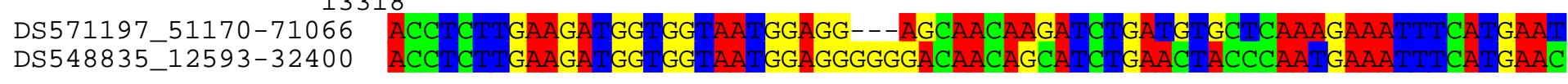
13387

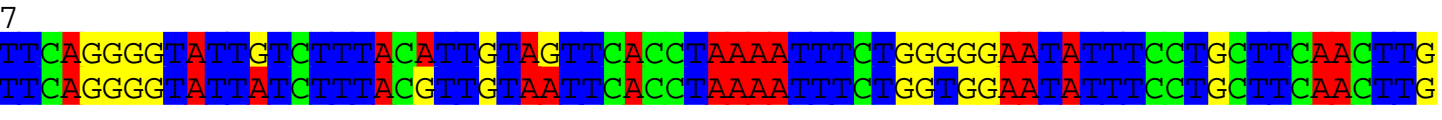

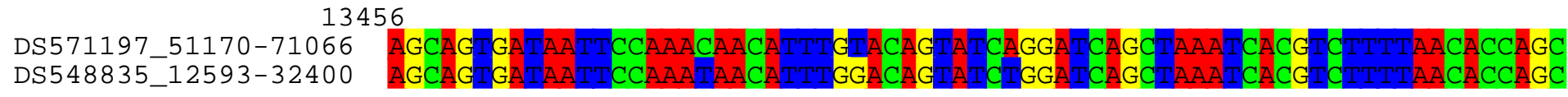

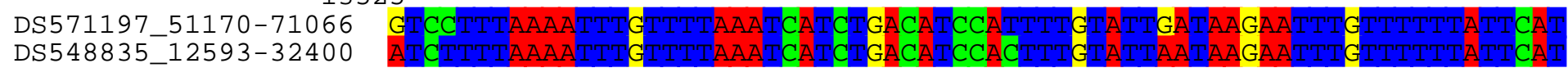




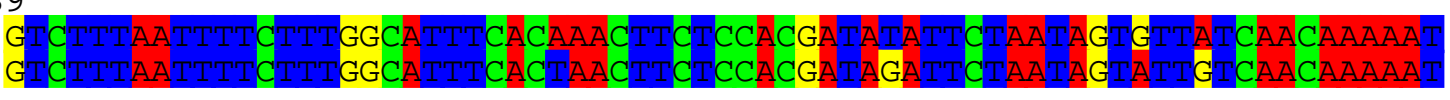




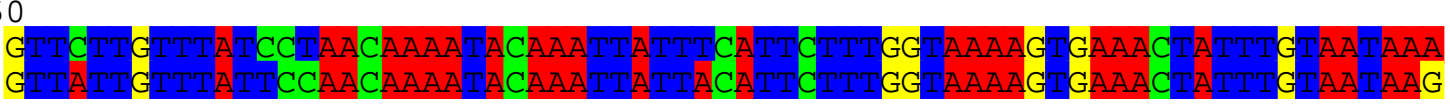
15319 DS571197_51170-71066 DS548835_12593-32400

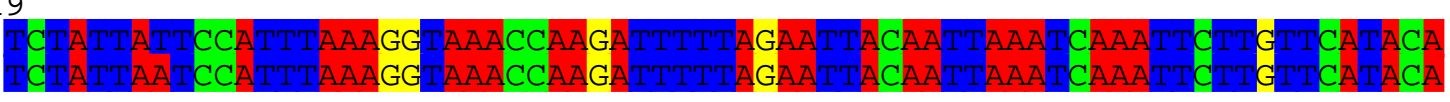

15388

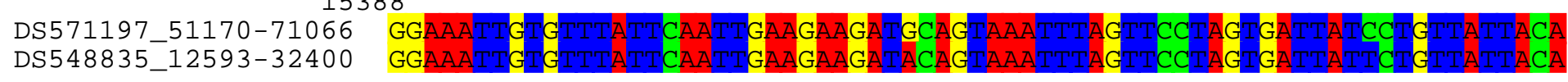
15457

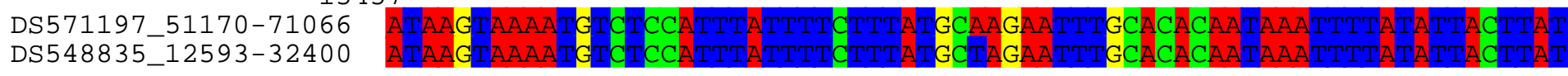

15526

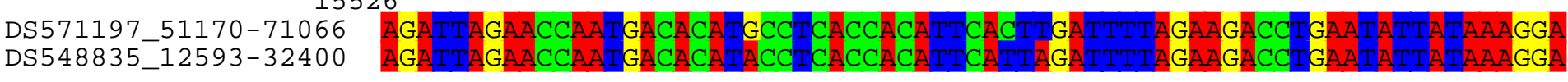
DS571197 $51170-71066$ A 159 DS548835_12593-32400

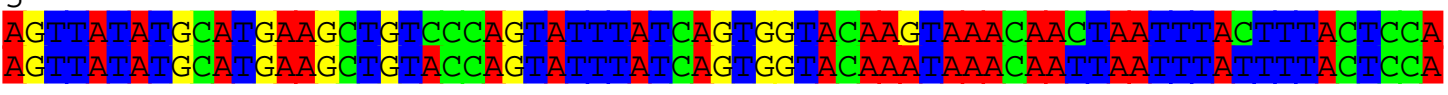
15664

DS571197_51170-71066 DS548835_12593-32400

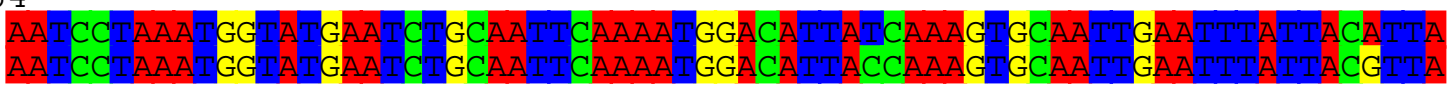
15733

DS571197_51170-71066 DS548835_12593-32400

\section{T'}

15802

DS571197_51170-71066

DS548835_12593-32400

AAAATTATTGAATTTATAAAAATGAAGAAATAGAACAAG GAAATATTATAACCA
AAAATTATIGAATTTATAAAAATGAAGAAACAGAACATG GAAATATTATAACTA TAAATGAT
CAAATGAT

15871

DS571197_51170-71066

DS548835_12593-32400

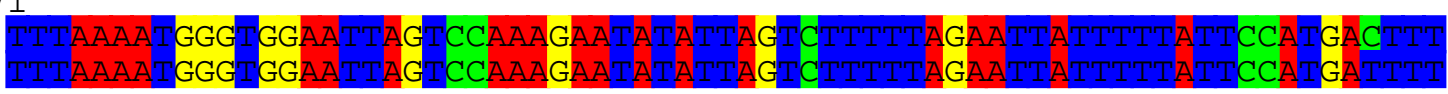

15940

DS571197_51170-71066

DS548835_12593-32400

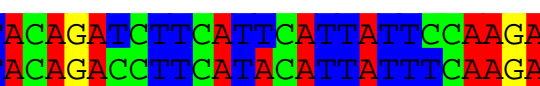

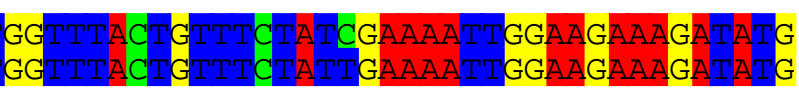

16009

DS571197_51170-71066

DS548835_12593-32400

GTTAAACATICCCTGGACAAC

GTAAAAATCTICAAGATGCTTTAGAAGAAGTATTAAAGAAAAA
GIAAAAAACIICAAGATGCTTIAGAAGAAGTIATAAAGAAAAA

16078

DS571197_51170-71066 AATACAGCATTAAAAAATAATAAAACTGTICCATTACAAAATCATTTTAATTCAGAAGGACATTCTACT
DS548835_12593-32400 AATACIGCATTAAAAAATAGAAAAGIICCATTACAAAAICGITTAAATCAGAAGGACATICIACA 16147

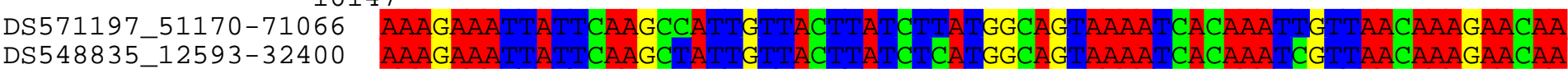
16216

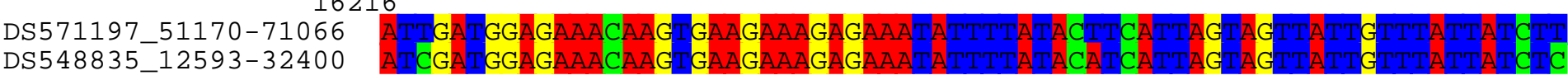

16285 
DS571197_51170-71066 CAAAAGAAAGAACTGGTGATAAAATAAAGTTATAAAAAAATIGAAGAACTTATIGAAGAAAAAATA
DS548835_12593-32400 CAAAAGAAAGAAATGGIATAGAAATAAATTIATAAAAAAATGAAGAACTATIGAAGAAAAAATA 16561

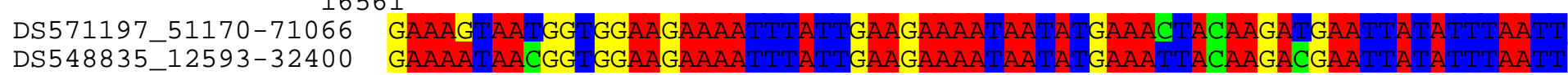
16630 DS571197_51170-71066 DS548835_12593-32400

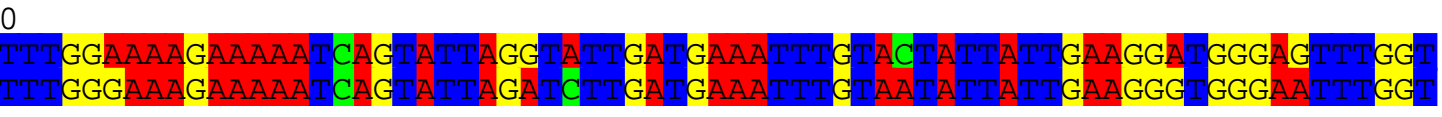
16699 DS571197_51170-71066 DS548835_12593-32400

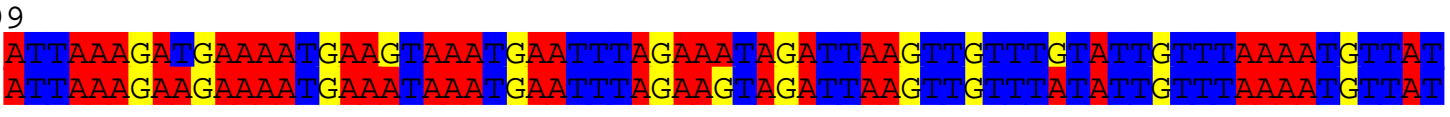

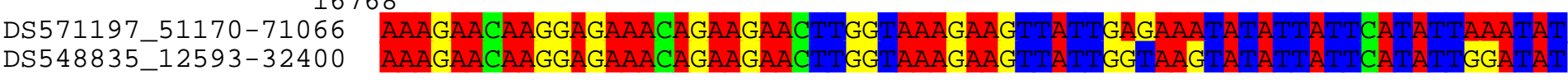
16837 DS571197_51170-71066 GCTTCAATAAATAATATTATATTAACTGATGAATATATTAAATCATTTATTGAAATATCAAACAAATAT
DS548835_12593-32400 GCCICAATAAAAATAICATATTAACIGAAGAATATIAAACGTIATIGAAATAICAAATAAATAI DS548835_12593-32400 GC

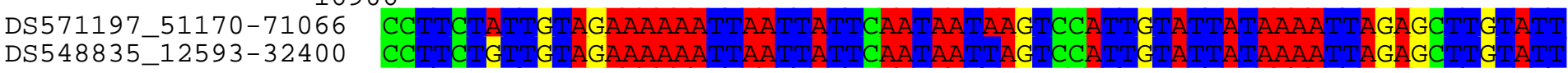
16975

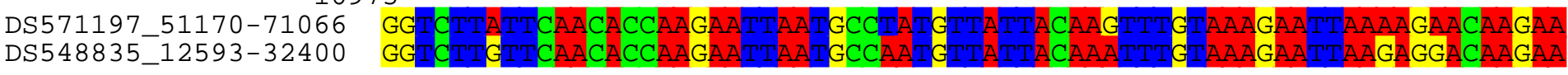
17044

DS571197_51170-71066 DS548835_12593-32400

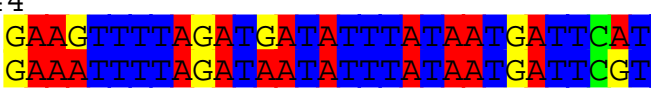
17113

DS571197_51170-71066

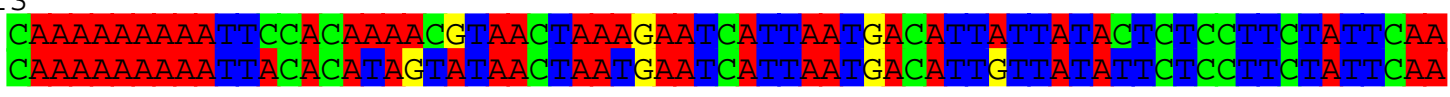
DS548835_12593-32400

17182

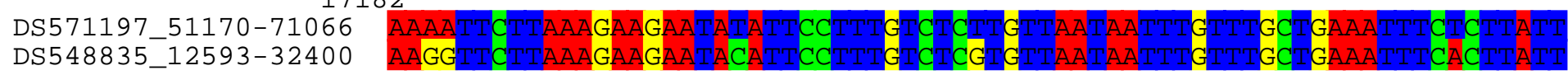
17251

DS571197_51170-71066 DS548835_12593-32400

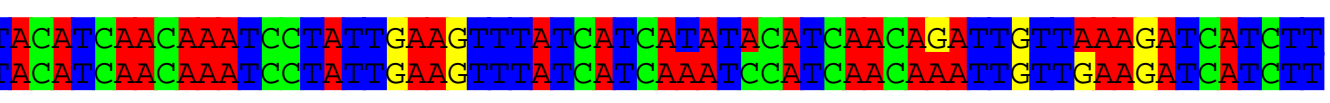
17320

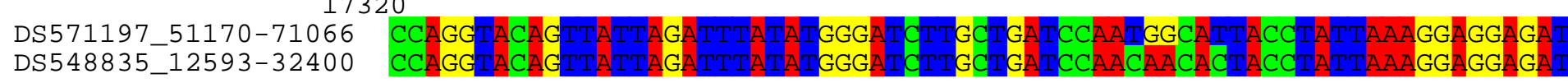
17389

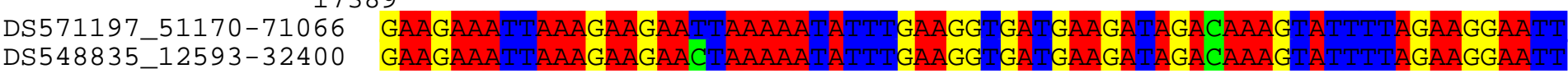

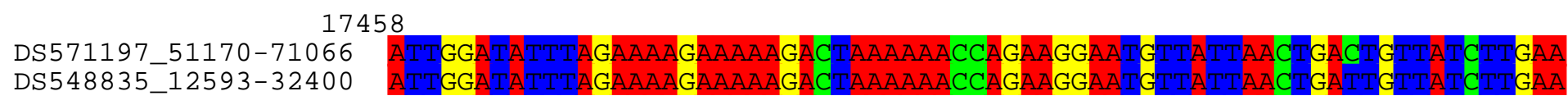
17527

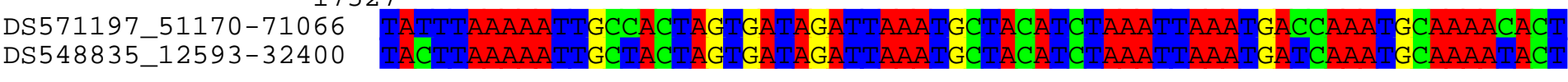
17596

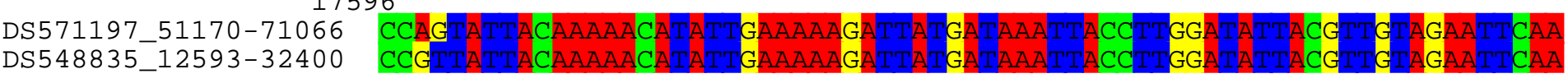
17665

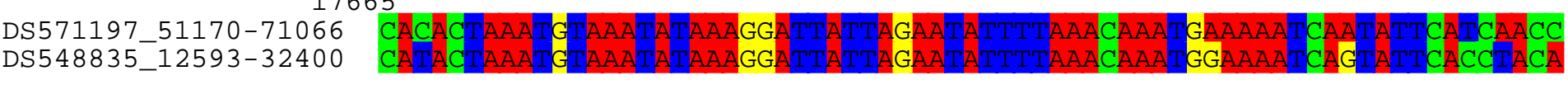
DS548835_12593-32400 C 


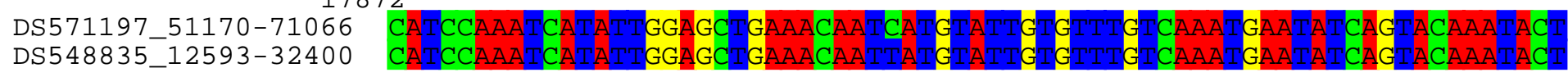
17941 DS571197_51170-71066 DS548835_12593-32400

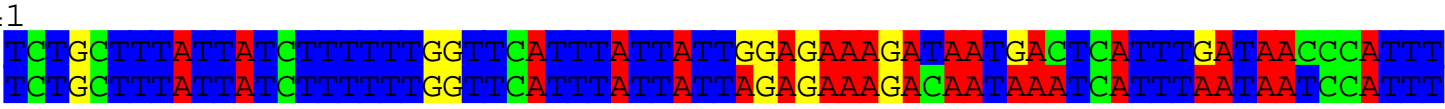

18010

DS571197_51170-71066 CCAATTCATTTGCATTAGAGTTATATAGTCGTTCTATCCTATTATTCCTTTAAAATTACTGAAGGT
DS548835_12593-32400 18079

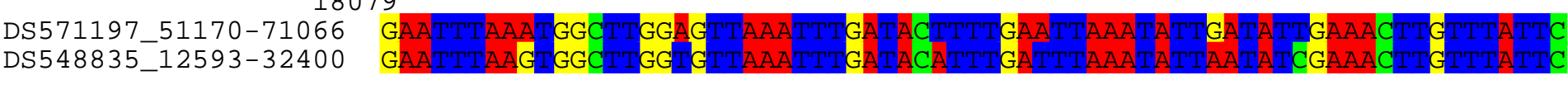
DS548835_12593-32400 G

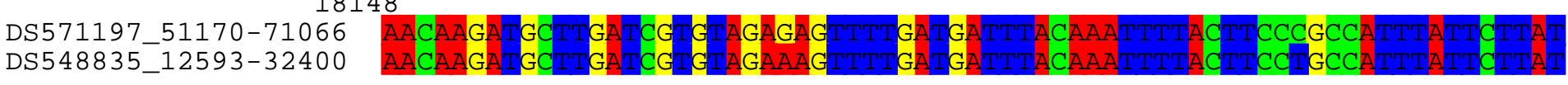

18217 DS571197_51170-71066 GCCAGAAAGTATTTCTACCTTTCTATTTATTCAAATGCTATTAAAGGATTAACTATAAATCTAATAAA
DS548835_12593-32400 GCTCGAAAATATTTCTACCTTTCTATTTATTCAAATGCTATTAAAGGATTAACCATAAATTCTAATAAA 18286

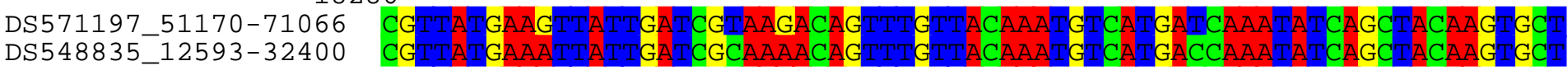
DS571197_51170-71066 1835 DS548835_12593-32400

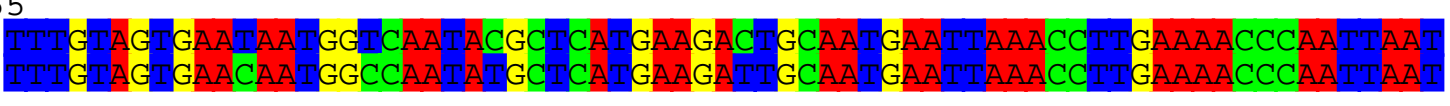
18424

DS571197_51170-71066 DS548835_12593-32400

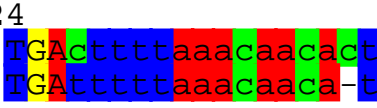

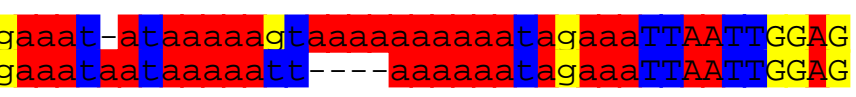
DS571197_51170-71066 AAGAGTGAACATATGCAATTGAGCAACACAAACTAATACTGTCATTGTTATACCAACTAATTTAAG
DS548835_12593-32400 AAGATIGAAACATATCAATTGCTGCAACACAAATTACTATIGICATAGTTATCCAACTAACTTTAAA 18562

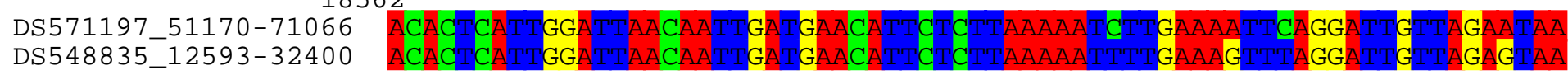
18631

DS571197_51170-71066 DS548835_12593-32400

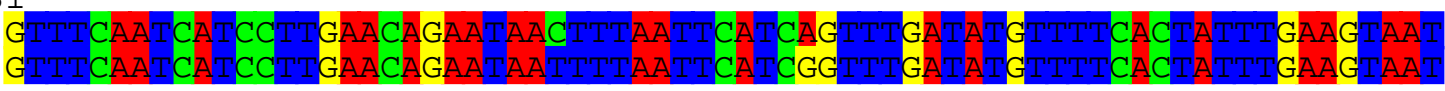

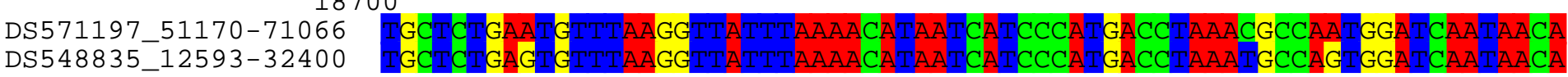
18769 DS571197_51170-71066 AGCATTTGTGAGCTAAATCCACTGATTCAGATAATCAAATCAAAGATTTAGATGGAACTTTCCT
DS548835_12593-32400 AGCATTGGGCAGCTAAGICAACTGATCAGATAACCCAAATTCAAAGATTTAGGTGGACTTTTCCI 18838

DS571197_51170-71066 DS548835_12593-32400

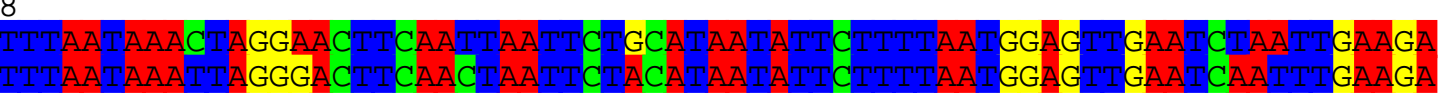

DS571197_51170-71066 GCATATGCTGCTTGGATGCTTTTAAAGAAACAGCCTGAGGTAATTTGTTTCTTAATAGCACTTAAT
DS548835_12593-32400 GTATATGCTGCTTGGATGCTTTTAATGAACAGCTTGAGGCGATTAATTTCTTAATAGCACTTAAT 18976

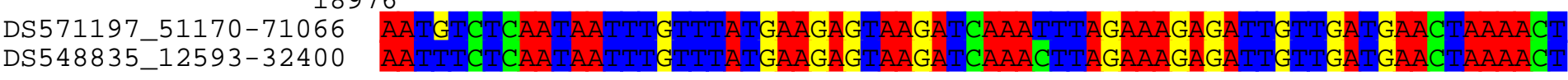
19045

DS571197_51170-71066 AAAGAATATCTTGTAAACTTCATGTTGATGAATAGATAAATCAAATGATATCAATTCTTCTTCA
DS548835_12593-32400 AAAGAAATACTTTGAAACTTCATGTTGAAGAATAGATAAATCAAATGAATATCATTTCTTCTTCA 19114

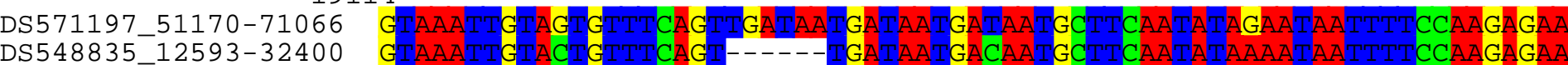


DS571197_51170-71066 DS548835_12593-32400

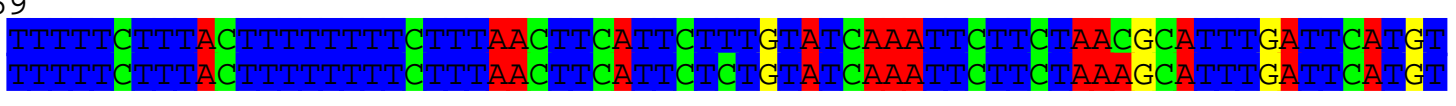

$$
19528
$$

DS571197_51170-71066 DS548835_12593-32400

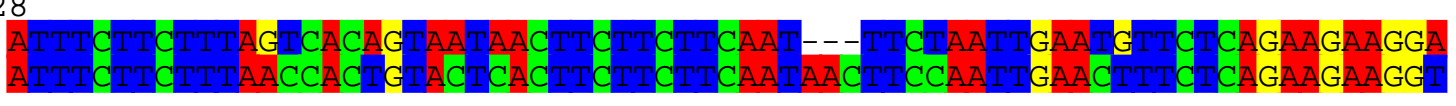

19597

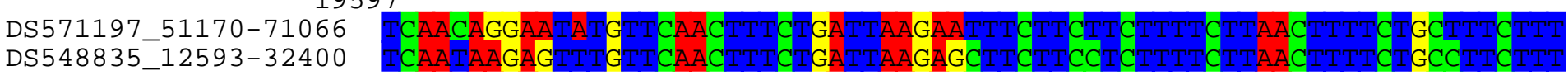

19666

DS571197_51170-71066 DS548835_12593-32400

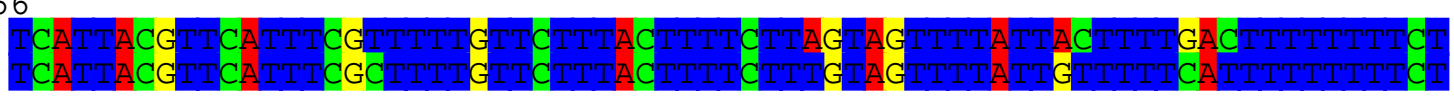

19735

DS571197_51170-71066 DS548835_12593-32400

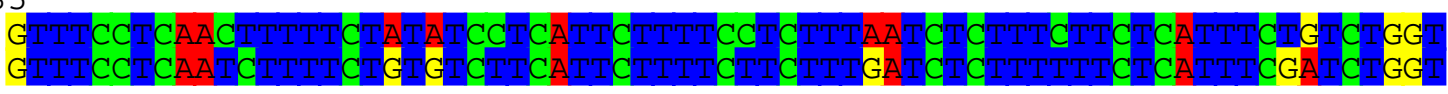
19804

DS571197_51170-71066 DS548835_12593-32400

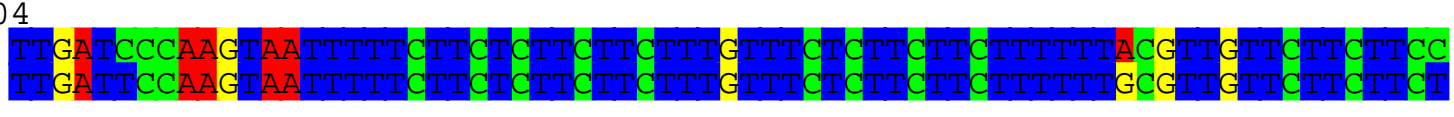
19873

DS571197_51170-71066 DS548835_12593-32400

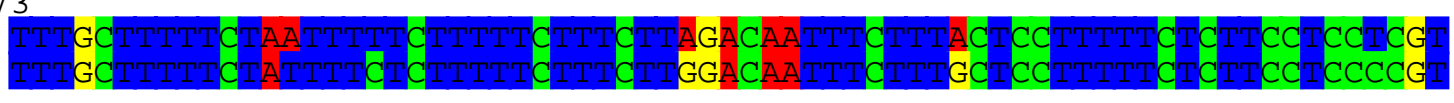
19942

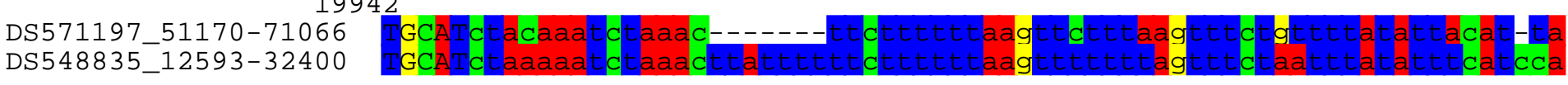
20011

DS571197_51170-71066

DS548835_12593-32400

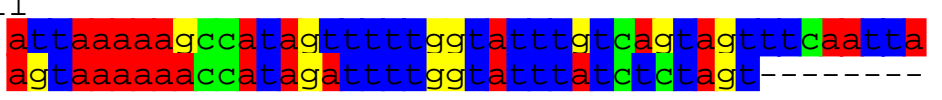

